\title{
Neural activation differences in amputees during imitation of intact versus amputee movements
}

\author{
William F. Cusack, Michael Cope, Sheryl Nathanson, Nikta Pirouz, Robert Kistenberg and \\ Lewis A. Wheaton*
}

School of Applied Physiology, Georgia Institute of Technology, Atlanta, GA, USA

\author{
Edited by: \\ John J. Foxe, Albert Einstein College \\ of Medicine, USA \\ Reviewed by: \\ Redmond O'Connell, Trinity College \\ Dublin, Ireland \\ Mario Tombini, Campus Bio-Medico \\ University of Rome, Italy \\ ${ }^{*}$ Correspondence: \\ Lewis A. Wheaton, School of \\ Applied Physiology, Georgia Institute \\ of Technology, 555 14th Street NW, \\ Atlanta, GA 30318, USA. \\ e-mail: lewis.wheaton@ \\ ap.gatech.edu
}

The mirror neuron system (MNS) has been attributed with increased activation in motor-related cortical areas upon viewing of another's actions. Recent work suggests that limb movements that are similar and dissimilar in appearance to that of the viewer equivalently activate the MNS. It is unclear if this result can be observed in the action encoding areas in amputees who use prosthetic devices. Intact subjects and upper extremity amputee prosthesis users were recruited to view video demonstrations of tools being used by an intact actor and a prosthetic device user. All subjects pantomimed the movements seen in the video while recording electroencephalography (EEG). Intact subjects showed equivalent left parietofrontal activity during imitation planning after watching the intact or prosthetic arm. Likewise, when prosthesis users imitated prosthesis demonstrations, typical left parietofrontal activation was observed. When prosthesis users imitated intact actors, an additional pattern was revealed which showed greater activity in right parietal and occipital regions that are associated with the mentalizing system. This change may be required for prosthesis users to plan imitation movements in which the limb states between the observed and the observer do not match. The finding that prosthesis users imitating other prosthesis users showed typical left parietofrontal activation suggests that these subjects engage normal planning related activity when they are able to imitate a limb matching their own. This result has significant implications on rehabilitation, as standard therapy involves training with an intact occupational therapist, which could necessitate atypical planning mechanisms in amputees when learning to use their prosthesis.

Keywords: mirror neuron, mentalizing, motor control, upper extremity, amputee, prosthesis, EEG, tool use

\section{INTRODUCTION}

Previous studies document that specific areas within the premotor, motor, and parietal cortices are activated when planning, executing and observing cognitive motor control tasks (Cattaneo and Rizzolatti, 2009). This network of areas has been defined as a "mirror neuron system (MNS)" that provides a mechanism by which we can understand, learn, and imitate the actions of others from our own perspective (Rizzolatti and Sinigaglia, 2010). The capacity of the MNS to activate the neuromotor network in the observer is seen in the concept of motor resonance. Under this concept, observation of an action drives an internal replication of that action in the motor system of the observer in a somatotopic manner (Buccino et al., 2001). This resonance can account for activation of the corticospinal pathways and task-specific muscles during action observation (Strafella and Paus, 2000; Funase et al., 2007; Alaerts et al., 2009).

Recent work shows that the MNS observation effects may be weakened when an individual witnesses a human movement performed by a virtual robotic actor with human-like kinematics. The sensorimotor areas were significantly deactivated when the subjects observed a human executing action that was robot-like (Tai et al., 2004; Shimada, 2010). This result suggests that the MNS may be preferentially engaged by limb movements that are similar in appearance and kinematic capabilities to that of the viewer. However, recent studies challenge this account (Rochat et al., 2010; van Elk et al., 2011).

Evidence also shows sensitivity of the MNS based on whether the observed action is possible for the observer to perform. It has been shown that observing non-conspecific actions that are not possible in humans activates bilateral visual responses instead of action encoding areas (Buccino et al., 2004). Similar bilateral and right hemispheric temporoparietal activations have been associated with a mentalizing system, which can be active when actions are observed that have no motor template in the observer (Van Overwalle and Baetens, 2009). The mentalizing system may be engaged in visual understanding of unfamiliar actions, rather than using typical action encoding (Wheatley et al., 2007). These results may suggest a different encoding mechanism for the observation of actions performed with dissimilar limb types.

The engagement of the mentalizing system during action observation and imitation may relate to the needs of amputees learning to use a prosthetic device. It has been demonstrated that fully functional adoption of prosthetic devices is low. Up to $33 \%$ of upper extremity amputees completely abandon their prostheses and cite the lack of usefulness in daily life as a reason. Further, $75 \%$ of amputees view their artificial limbs as functional aesthetic 
devices whose principal function is to restore symmetry to their appearance (Datta et al., 2004). A lack of knowledge in how to use the device properly has been suggested as a reason for such high prosthesis user dissatisfaction (Kejlaa, 1993; Dudkiewicz et al., 2004). This high level of dissatisfaction leads many amputees to wear their prosthetic device passively (Biddiss and Chau, 2007a,b). Knowledge gained regarding the neural systems activated by amputees while performing motor tasks demonstrated by similar and dissimilar arm types may provide insight into their action planning mechanisms. If neural activation differs between intact subjects and amputees, this could reveal unique action planning strategies in amputees. These atypical strategies may relate to the challenges experienced by amputees while developing action representations that incorporate a prosthesis.

The majority of research regarding action encoding and planning mechanisms has been performed in intact and healthy subjects. The aim of the present work is to investigate whether or not neural activations during planning and execution in amputee prosthesis users vary as a function of the type of limb they see performing an action. This was investigated by instructing both intact subjects and prosthesis users to imitate tool use movements observed in video demonstrations featuring an intact actor and prosthesis user. Electroencephalography (EEG) was recorded during the movement trials. This method allowed for subjects to sit in a comfortable upright position while performing the tasks in a naturalistic manner; an advantage over functional magnetic resonance imaging (fMRI) techniques, which require subjects to lay prone in a confined space.

Prior work using EEG to study imitation of tool-use tasks has revealed differential cortical activity based on the familiarity of the action. Imitation pantomime of familiar actions elicited greater activity in the parietofrontal cortical regions during movement planning, while imitation of unfamiliar actions elicited greater activity in the temporoparietal-occipital cortical regions (Mizelle et al., 2011). This finding suggests that the relative engagement of these two action encoding systems is a function of the level of motor resonance between the subject's movement repertoire and the action to be imitated. The use of EEG has also been successful in establishing networks that relate to understanding action goals (left hemispheric) and action intent (right hemispheric) (Ortigue et al., 2010). These results corroborate fMRI findings showing greater temporoparietal-occipital activation upon planning the pantomime of unfamiliar tool-use actions (Vingerhoets, 2008; Quallo et al., 2009; Vingerhoets et al., 2011). To our knowledge, the present work is the first to investigate tool-use imitation action encoding in upper extremity amputees.

In this present study, we will utilize a cued EEG paradigm to prompt the planning and performance of a tool use pantomime in amputees and intact subjects. These paradigms are often advantageous over self-paced paradigms as they more clearly discern movement related potentials in the planning and execution phases (Jankelowitz and Colebatch, 2002). This concept has been demonstrated in prior work using EEG and fMRI to study praxis movement in healthy subjects (Fridman et al., 2006; Bohlhalter et al., 2009).

We hypothesized that the neural activations of intact subjects would not be affected by the video demonstration type, but that those of the prosthesis users would be. Specifically, prosthesis users imitating another prosthesis user would activate the left parietofrontal network, consistent with tool use neural activity with an existing motor template that should exhibit stronger resonance with the action. Contrastingly, prosthesis users imitating an intact individual would activate the right parietooccipital network, in addition to the typical parietofrontal regions. This would reflect the increased visuospatial demands of imitating the movements of a dissimilar limb without a readily available motor template.

\section{MATERIALS AND METHODS SUBJECTS}

Ten right-handed intact subjects were recruited for this study (four female, six male, mean age: $24.8 \pm 3.3$ years, range: $23-34$ years). Six upper extremity amputee prosthesis users were also recruited (two female, four male, mean age: $44.3 \pm 9.9$ years, range: 33-59 years). Signed informed consent was acquired from all subjects according to the procedures set forth by the Institutional Review Board at The Georgia Institute of Technology. The Edinburgh Handedness Inventory (Oldfield, 1971) was use to confirm the handedness of the intact subjects and, in the case of amputees, recalled handedness prior to their amputation. Amputee subjects reported wearing their prosthetic devices an average of $4.4 \pm 3.6 \mathrm{~h}$ /day. Average psychosocial adjustment of the amputees to their prostheses, as assessed by the TAPES survey (Desmond and Maclachlan, 2005), was calculated to be $51.6 \pm 9.0$ (on a scale from 14-70, with higher values indicating greater adjustment). Before being recruited into the protocol, all subjects were screened for the presence of any other neurologic factors, including (but not limited to) traumatic brain injury, stroke, or concussion. The presence of phantom limb syndrome/pain was an exclusion criterion for the amputee subjects. The amputee population was made up of persons who had lost their limb due to occupational and recreational accidents with no brain trauma. Demographic and clinically relevant information for the prosthesis users is presented in Table 1. Notably, with the exception of one especially chronic amputee, the amputee subjects had experienced their amputations within a similar time frame (0.6-3.5 years) and they had also been using their current prosthetic devices for a similar time frame (0.3-2.3 years) (Table 1).

\section{PROCEDURE}

Subjects were fitted with a 58-channel EEG cap (Electrocap, Eaton, $\mathrm{OH}$ ) that recorded scalp potential activity ( $1 \mathrm{kHz}$ sampling rate, filtered at $\mathrm{DC}-100 \mathrm{~Hz}$ ) via the Synamps two data acquisition system (Compumedics Neuroscan, Charlotte, NC). For analysis, EEG data was further pass-band filtered from DC-30 Hz. Electrooculography was recorded in two locations near the left eye to monitor eye blinks and movements. Surface electromyographical (EMG) data ( $1 \mathrm{kHz}$ sampling rate, filtered at $20-100 \mathrm{~Hz})$ of the biceps brachii, triceps brachii, anterior deltoid, and posterior deltoid were recorded to inform of the onset of volitional movement.

Subjects were seated $1.5 \mathrm{~m}$ away from a computer screen that displayed videos of tool use movements followed by written 
Table 1 | Demographic and clinically relevant information for the prosthesis users.

\begin{tabular}{|c|c|c|c|c|c|c|c|c|c|c|c|c|c|}
\hline & Sex & $\begin{array}{l}\text { Age } \\
\text { (yrs) }\end{array}$ & $\begin{array}{l}\text { Hand } \\
\text { dom. }\end{array}$ & $\begin{array}{l}\text { Amp. } \\
\text { cause }\end{array}$ & Level & $\begin{array}{l}\text { Amp. } \\
\text { side }\end{array}$ & $\begin{array}{l}\text { Years } \\
\text { since amp. }\end{array}$ & $\begin{array}{l}\text { Years since } \\
1^{\text {st }} \mathrm{PX}\end{array}$ & $\begin{array}{l}\text { Years with } \\
\text { current PX }\end{array}$ & $\begin{array}{l}\text { Power } \\
\text { type }\end{array}$ & TD & $\begin{array}{l}\text { PX use } \\
\text { (h/day) }\end{array}$ & PAS \\
\hline Pro1 & $\mathrm{F}$ & 53 & $\mathrm{R}$ & TRAU & $\mathrm{TR}$ & $L$ & 17.0 & 16.8 & 16.8 & Body & Hook & 0 & 52 \\
\hline Pro3 & $M$ & 33 & $\mathrm{R}$ & TRAU & $\mathrm{TR}$ & $\mathrm{R}$ & 0.8 & 0.6 & 0.3 & Myo & Hook & 10 & 39 \\
\hline Pro4 & $M$ & 44 & $\mathrm{R}$ & TRAU & $\mathrm{TR}$ & $\mathrm{R}$ & 2.5 & 2.3 & 2.3 & Body & Hook & NR & NR \\
\hline Pro5 & $M$ & 38 & $\mathrm{R}$ & TRAU & ED & $R$ & 2.5 & 2.3 & 1.3 & Body & Hook & 3 & 63 \\
\hline Mean \pm SD & - & $44.3 \pm 9.9$ & - & - & - & - & $5.1 \pm 6.0$ & $4.6 \pm 6.1$ & $3.8 \pm 6.4$ & - & - & $4.4 \pm 3.6$ & $51.6 \pm 9.0$ \\
\hline
\end{tabular}

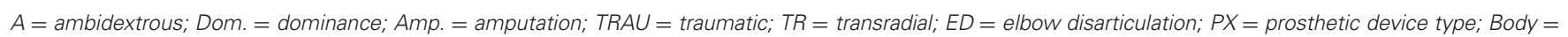

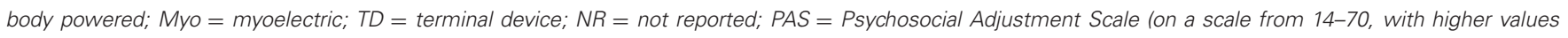
indicating greater adjustment).

directions regarding the movement tasks to be performed. All subjects viewed video demonstrations of common tools being used by both an intact actor and an actor wearing a body powered prosthetic device. In the case of prosthesis users, the terminal device featured in the video matched that of the subject. A Model 5X Hook (Hosmer, Campbell, CA) was used for those subjects with a voluntary opening, split hook type terminal device (Figure 1A) while a simple articulated anthropomorphic hand was used for those subjects with a hand-type terminal device (Figure 1B). Intact subjects only viewed the Model 5X Hook terminal device.

After the presentation of each video, all subjects were instructed to imitate the action they had seen in the video by pantomime. Intact subjects performed the task with their dominant arm and amputees used their prosthetic device. The prosthesis users were not required to perform active prehension with their terminal devices in order to successfully pantomime the observed movements. There were four experimental groups: intact subjects

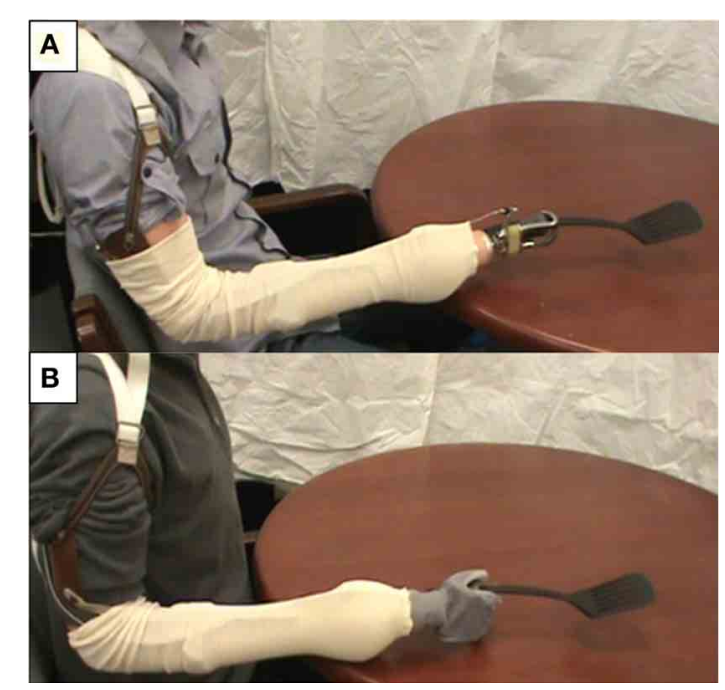

FIGURE 1 | Prosthetic limb terminal devices used in tool use video demonstrations: Hosmer Model 5X Hook (A) and simple articulated anthropomorphic hand (B). imitating an intact actor (Int-Int), intact subjects imitating a prosthesis user (Int-Pro), prosthesis users imitating an intact actor (Pro-Int), and prosthesis users imitating a prosthesis user (Pro-Pro). There were six tool use tasks performed in the video demonstrations. In three tasks, an intact actor performed switching a light switch, drinking from a water bottle, and checking boxes with a pen. In the remaining three tasks a prosthesis user performed flipping a pancake with a spatula, shaking spices out of a dispenser, and turning a key in a lock. The videos were presented in an alternating order such that each successive video displayed a demonstrator arm type different than the previous. Each video was shown for $60 \mathrm{~s}$ and contained exactly six movement repetitions.

After watching each video, subjects performed tool use motor tasks that were visually cued using Stim (Compumedics Neuroscan, Charlotte, NC). Subjects fixated on a white cross for a randomly determined baseline period of 4.0-6.0 s. Subjects were then instructed to remain motionless and begin planning for the movement upon seeing a "Get Ready!" cue for $1.0 \mathrm{~s}$. Immediately afterward a final cue appeared commanding them to "Move!" which remained on the screen for $4.0 \mathrm{~s}$. During this period, subjects were instructed to imitate the movement they observed in the video by pantomiming the action. For technical reasons, it was not possible for each movement cue to be preceded by a repetition of the video demonstration. This cued movement sequence was repeated 50 times for each of the six demonstrations, for a total of 300 movements per data collection. All subjects were periodically allowed rest sessions between movement trials to mitigate effects related to fatigue. Presentation of the "Move!" cue is aligned with the zero point on the timeline. The $1.0 \mathrm{~s}$ preceding this cue is referred to henceforth as the movement planning phase while the period of time after this cue is referred to as the movement execution phase. To characterize the movement quality of the two subject populations imitating the tool demonstrations, all movements were rated by a single evaluator according to a pantomime recognition scale (PRS) (see Appendix, maximum score 4) (Wheaton et al., 2008; Bohlhalter et al., 2009).

\section{DATA RECORDING AND ANALYSIS}

Using Scan4.5 (Compumedics Neuroscan, Charlotte, NC), continuous EEG data were epoched to $3.1 \mathrm{~s}$ epochs centered on 
the presentation of the "Move!" cue ( -1.6 to $1.5 \mathrm{~s})$ and linear detrended. Baseline correction between -1.6 and $-1.1 \mathrm{~s}$ relative to the "Move!" cue was then performed. A combination of artifact averaging and regression analysis was employed to remove ocular artifacts (Semlitsch et al., 1986). A final visual inspection was performed and any epoch containing data that was outside a threshold range of -100 to $100 \mu \mathrm{V}$ was rejected. Individual subject data was then averaged across individual tool type and grouped into the four experimental conditions described previously. The grouping of unlike tools together was designed intentionally to elicit general tool related activation in the parietofrontal cortical regions (Moll et al., 2000; Hermsdorfer et al., 2007; Bohlhalter et al., 2009; Wheaton et al., 2009). This network has been shown to be responsible for action encoding of general tool-use, rather than for processing specific tool information (Jeannerod et al., 1995; Johnson-Frey, 2004; Mizelle and Wheaton, 2010). The generalization of the specific tools observed in the videos allowed the investigation to focus on the effect of the arm type being imitated during movement planning.

Statistical analysis and plotting were then performed using MATLAB software (The MathWorks, Natick, MA). All epochs were averaged into $100 \mathrm{~ms}$ timebins and further grouped into the regions of interest that were defined in the left and right premotor areas (LPM: F3, F1, C5A, C3A, C1A; RPM: F4, F2, C6A, C4A, C2A), left and right motor areas (LM: C5, C3, C1; RM: C6, C4, C2), left and right parietal areas (LP: TCP1, P5, P3, P1, P3P; RP: TCP2, P6, P4, P2, P4P), and occipital area (OCC: O1, OZ, O2) (Wheaton et al., 2005b). To enhance our ability to capture the timing of differences in the neural signal, t-tests were performed across $100 \mathrm{~ms}$ timebins from the onset of the planning cue through $1.5 \mathrm{~s}$ after the move cue. Four statistical comparisons were performed in this analysis. The first pair of statistical analyses evaluated the effect of the video demonstration type within each subject type (Int-Int vs. Int-Pro; Pro-Pro vs. Pro-Int). The next pair of statistical analyses evaluated the effect of subject arm type within each video demonstration type (Int-Int vs. Pro-Int; Int-Pro vs. Pro-Pro). This latter set of comparisons is particularly salient due to the consistency of the tool movements being imitated within each subject type. The threshold for statistical significance was held at $\alpha=0.001$ for all comparisons. To avoid misinterpretation of spurious cortical activity differences and to ensure that the differences observed are not transient, statistically significant differences between groups must be maintained for four consecutive timebins (400 ms).
Filtered EMG data were averaged together within each subject and experimental condition. For each set of movements, baseline EMG activity was defined as the mean activity of the $500 \mathrm{~ms}$ preceding the movement cue. EMG onset occurred once the activity surpassed two standard deviations of the baseline mean for a period of at least $25 \mathrm{~ms}$. Statistical t-tests were performed within each muscle type to determine if the intact subjects and prosthesis users initiated EMG onset at different time intervals when performing imitations of the same tool types. The threshold for statistical significance was held at $\alpha=0.001$. Table 2 shows the average EMG onset times and $p$-values for each of the four muscles recorded for the following comparisons: Int-Int vs. Pro-Int and Int-Pro vs. Pro-Pro. Further, in order to investigate whether the EMG onsets were affected by either of the experimental variables, multivariate ANOVAs were performed with subject group (2) and muscle type (4) as factors. In order to control for the tool use movements being imitated, separate ANOVAs were performed on the pairs of groups that imitated intact actors (Int-Int; Pro-Int) and prosthesis users (Int-Pro; Pro-Pro). The threshold for statistical significance was held at $\alpha=0.05$ for all ANOVAs.

\section{RESULTS}

\section{EMG AND MOVEMENT OUALITY}

The results show that there were no differences between the overall EMG onset times between the intact subjects and prosthesis users within each muscle (Table 2). Further, an ANOVA comparing muscle activation onset for Int-Pro vs. Pro-Pro did not show a main effect for specific muscle $(F=2.67, p=0.06)$, subject arm type $(F=0.22, p=0.64)$, or an interaction between the two factors $(F=0.31, p=0.82)$. However, an ANOVA analysis comparing Int-Int vs. Pro-Int revealed a main effect on EMG onset for specific muscle $(F=2.38, p=0.04)$, but not for subject arm type $(F=3.62, p=0.06)$, or an interaction between the two factors $(F=1.15, p=0.34)$. While not statistically significant, a difference in EMG onset order was observed. The Int-Int, Int-Pro and Pro-Pro groups show a common sequence of EMG onset in the following order: anterior deltoid, biceps brachii, triceps brachii, posterior deltoid. The Pro-Int group shows the following unique pattern of EMG onset: biceps brachii, anterior deltoid, posterior deltoid, and triceps brachii.

For the intact video imitations, intact subjects received significantly higher PRS scores (Int-Int, $4.0 \pm 0.0$ ) than the prosthesis users (Pro-Int, $3.35 \pm 0.58)(p<0.001)$. Similarly, for the prosthesis video imitations, intact subjects received significantly

Table 2 | EMG onset data.

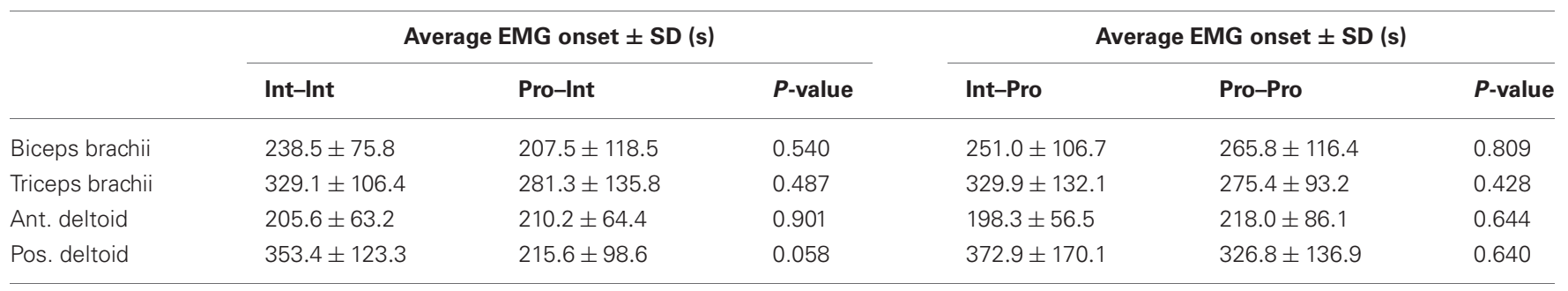

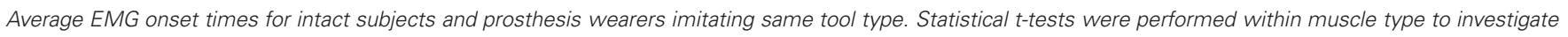
if EMG onset was different between the two subject populations. Significance threshold was held at $\alpha=0.001$. 
higher PRS scores (Int-Pro, $4.0 \pm 0.0)$ that the prosthesis users (Pro-Pro, $2.83 \pm 0.47)(p<0.001)$.

\section{NEURAL OUTCOMES}

\section{Effect of video demonstration arm type}

Figure 2 shows the grand-averaged voltage plots for the comparison between intact subjects imitating both intact actor and prosthesis user demonstrations (Int-Int vs. Int-Pro). There was no effect of demonstration arm type on the imitation neural activations of intact subjects during movement planning or execution ( $\alpha=0.001)$. Both groups exhibited the same pattern of high left parietal and mesial frontal negativity during movement planning and motor region negativity during movement execution as shown in Figure 3.

In contrast to the intact group, Figure 4 shows that there is an effect of demonstration arm type on the neural activations of prosthesis users (Pro-Pro vs. Pro-Int). The Pro-Int group (arm type mismatched) showed significantly greater positivity $(p<0.001)$ during movement planning in the right parietal ( -400 to $0 \mathrm{~ms})$ and occipital regions ( -400 to $0 \mathrm{~ms})$.

\section{Effect of subject arm type}

To establish that differences in subject arm type are the principal contributors to the observed planning related cortical activation patterns, the next two comparisons were performed between intact subjects and prosthesis users who were both prompted by the same set of tool use movements. Figure 5 demonstrates that there was an effect of subject arm type when both groups imitated the intact video demonstrations (Int-Int vs. Pro-Int). The Pro-Int group showed significantly greater positivity $(\mathrm{p}<0.001)$ during movement planning and early execution in the bilateral parietal ( -50 to $100 \mathrm{~ms})$ and occipital regions ( -500 to $0 \mathrm{~ms}$ ).

Figure 6 reveals that the arm type mismatch planning differences are mitigated when a prosthesis user imitates another prosthesis user (Int-Pro vs. Pro-Pro). In this comparison, amputees who imitated a matched arm type showed no significant neural
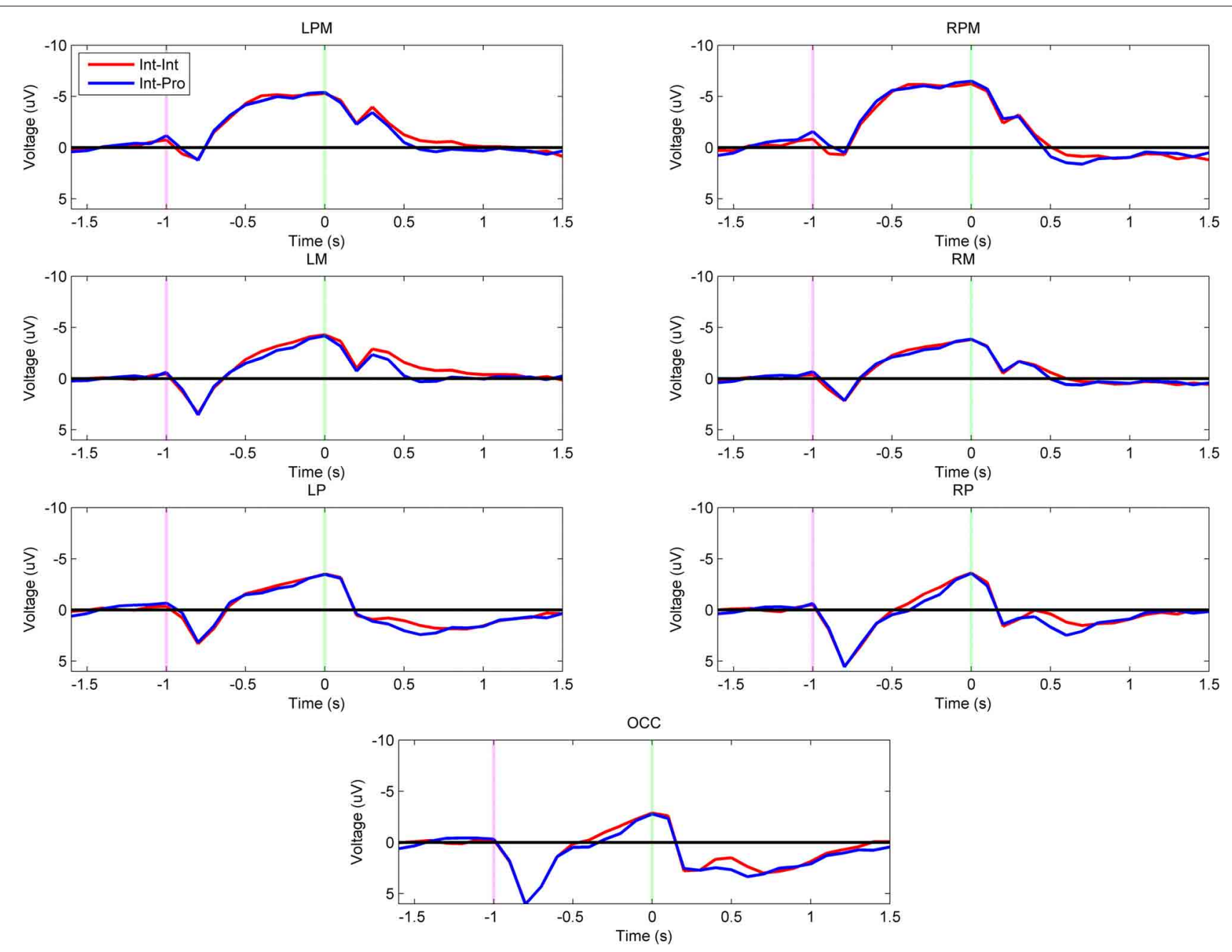

FIGURE 2 | Grand-averaged region-level voltage plots for the Int-Int (red) and Int-Pro (blue) groups. The planning cue is marked with a pink dotted line at $-1.0 \mathrm{~s}$ and the execution cue is marked with a green dotted line at $0.0 \mathrm{~s}$. Time bin voltage values that are statistically different between the two groups are marked with asterisks. 


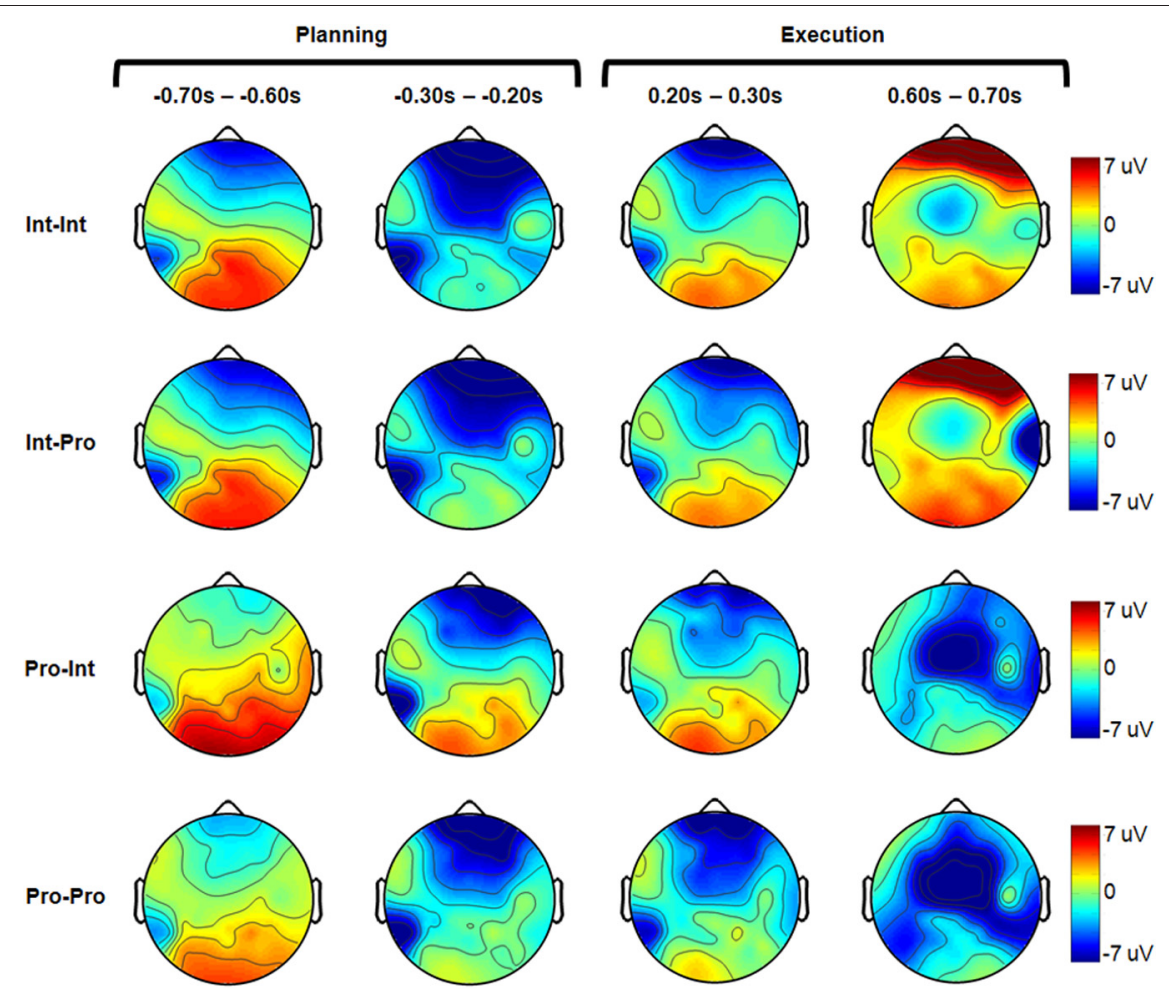

FIGURE 3 | Grand-averaged electrode headplots for all experimental conditions. For each condition, four representative 100 ms timebins are shown. The planning and execution phases are marked above.

differences from intact subjects during the movement planning phase $(\alpha=0.001)$. This pattern was also apparent when comparing the Int-Pro vs. Pro-Pro group headplots in Figure 3. Differences between these two groups were seen during execution. Compared to the intact subjects, the prosthesis users showed greater negativity $(p<0.001)$ in the bilateral premotor (500-1000 ms), left motor (500-900 ms), left parietal (400-1000 ms), and right parietal regions (400-900 ms) during execution (Figure 6).

\section{DISCUSSION}

Intact subjects and upper extremity amputee prosthesis users were recruited to view and imitate video demonstrations of tools being used by an intact actor and a prosthetic device user. The intact subjects showed equivalent left parietal and mesial frontal activation for imitating both the intact or prosthetic limb. However, when prosthesis users imitated intact subjects, greater right parietal and occipital activation during planning was observed in addition to parietofrontal activation. Prosthesis users who imitated other prosthesis users showed only the typical left parietal and mesial frontal activation. This finding suggests that prosthesis users can engage the anticipated left hemispheric planning related activity and disengage the parietooccipital system when they imitate a limb state that matches their own. The limb imitation effects seen in the amputees suggest the additional involvement of unique planning mechanisms while using their prosthetic device. This result has implications on how device operation is conveyed to amputees during rehabilitation.

In this study, the interpretation of execution phase cortical activity comparisons across groups is limited by a number of factors. Most significantly, amputees require a different number, combination, and activation level of muscles in order to complete the movement task with their residual limb (Schabowsky et al., 2008; Velliste et al., 2008; Metzger et al., 2010). The altered kinematics and kinetics of the limb-prosthesis system also necessitate the development of novel motor control strategies that yield unique movement characteristics in amputees (Bouwsema et al., 2010; Losier et al., 2011). Such physiological and functional variations may account for the significantly lower PRS scores received by prosthesis users compared to intact subjects. However, a limitation of the current study is the lack of a direct link between the observed cortical activation changes and the explicit motor performance of the tasks. The effect of atypical cortical action encoding strategies on motor task performance is presently being investigated.

With the exception of one especially chronic amputee, the amputee subjects had experienced their amputations within a similar time frame. Typically, significant time (several months) will elapse between the amputation and the first delivery of a prosthesis (Reinkensmeyer et al., 2012). This time delay is due to the slow progression of the healing process post-amputation. Once sufficiently recovered from the surgery, substantial time is also required in order to properly customize and fit the device 


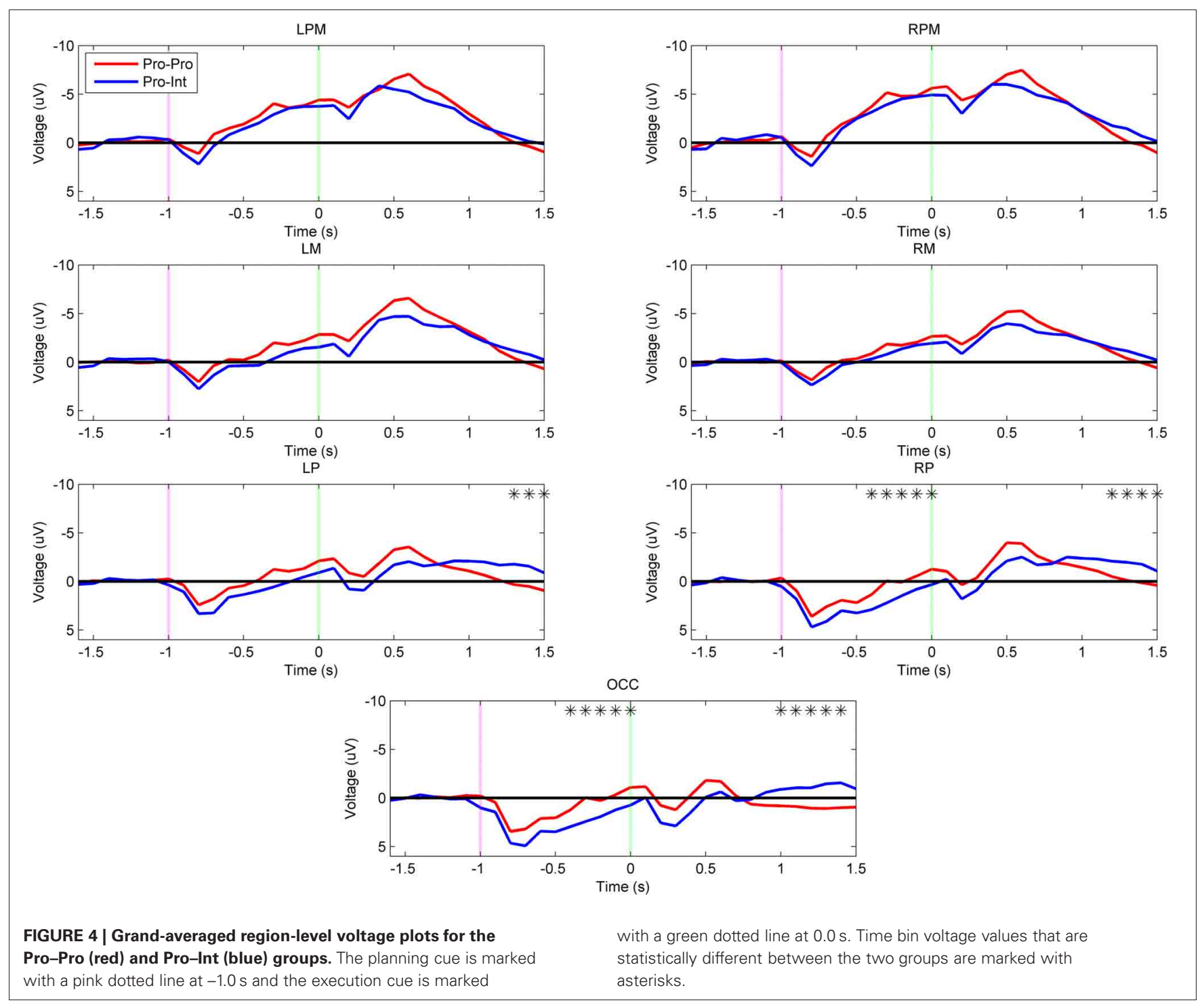

to the user. Therefore, the subjects recruited for this work are considered to be in the early stages of their prosthesis use. Acquisition of amputees at a more acute level is impractical. Further, many of the amputee subjects had recently received new prosthetic devices which would require an additional adjustment period for functional use. Importantly, subjects were recruited that were no longer experiencing phantom limb syndrome or pain. These symptoms can persist for months or years following the initial wound healing (Ramachandran and Hirstein, 1998; Jackson, 2004). Thus, it was necessary to recruit from a relatively chronic amputee population in order to meet these exclusion criteria. The characterization of the time course of amputee adjustment to their prostheses is currently being investigated. Additionally, given the preliminary scope of this study, the low number of amputee subjects is justifiable as we were seeking to select a very selective, but relatively homogenous cohort of amputees. The number of subjects in the current study is comparable to the majority of research on the upper extremity amputee population (Montoya et al., 1998; Schaefer et al., 2002; Karl et al., 2004a,b).
Further, sets of unlike tool demonstrations were grouped together into two categories: those demonstrated in the videos by an intact actor and those demonstrated by a prosthesis user. For example, in the comparison of Int-Int vs. Int-Pro, the intact subjects in each group are imitating different tool use movements. This logic is common for analysis for this task type, as the parietofrontal tool network seems to encode actions for general tool use rather than for specific tools (Jeannerod et al., 1995; JohnsonFrey, 2004). As such, the interpretation of execution phase cortical activity is difficult as a result of the muscle activations and joint coordination required for each of the unique tool-use tasks. Notably, though, in the example comparison cited above (Int-Int vs. Int-Pro), the cortical activations during execution were statistically equivalent despite the different tool movements being performed. Therefore, it is reasonable to state that the differences observed in execution phase cortical activations for the Pro-Int vs. Pro-Pro comparison are most likely due to the differences in video demonstration arm type and not the type of tool movement imitated. Nonetheless, for the reasons stated above, the focus of 


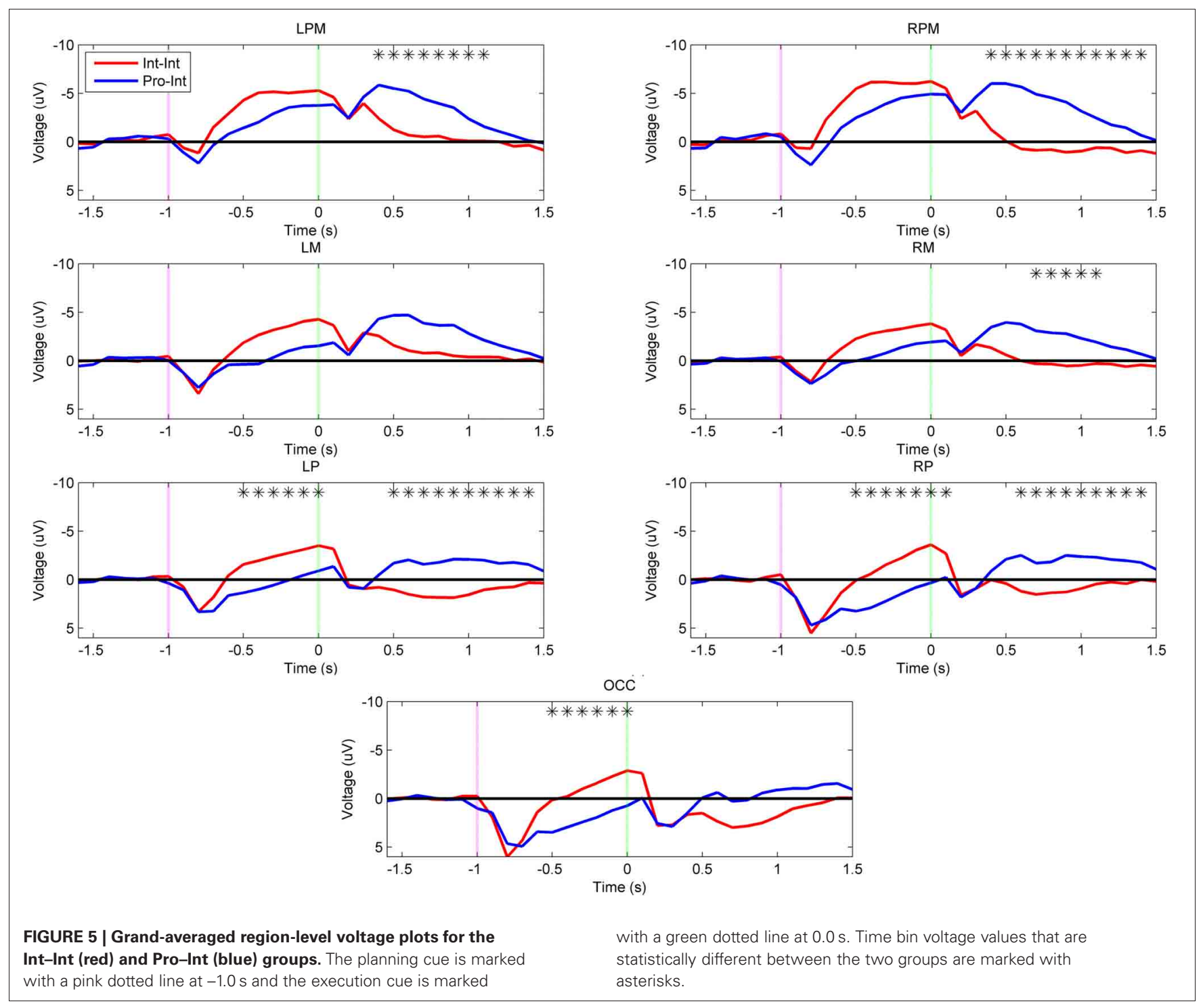

this discussion is on the planning phase cortical activity prior to movement onset.

\section{EMG AND MOVEMENT QUALITY}

The EMG onset data suggest that all experimental groups initiated EMG onset after a consistent period of time following the movement cue. While not statistically significant, a potential difference in muscle recruitment order was observed. Despite the differences in tool imitation movements, both intact subject groups (Int-Int, Int-Pro) reveal a consistent order of muscle activation. This pattern proceeds with a pattern of shoulder flexion (anterior deltoid), elbow flexion (biceps brachii), elbow extension (triceps brachii), and shoulder extension (posterior deltoid). For prosthesis users, this pattern is observed only when they imitate other prosthesis users. Otherwise, when a prosthesis user imitates an intact actor, a different pattern of EMG onset emerges: flexion of the elbow (biceps brachii), flexion of the shoulder (anterior deltoid), extension of the shoulder (posterior deltoid), and extension of the elbow (triceps brachii). These observations potentially

suggest that the kinematics and kinetics of tool use movement in prosthesis users may be influenced by the type of limb being imitated. Understanding the motor control and biomechanics of movement during task execution in prosthesis users is a focus of ongoing studies. Utilizing more sensitive techniques such as intramuscular EMG and kinematic assessments may improve our interpretation of these outcomes.

\section{ACTION PLANNING IN INTACT SUBJECTS}

Intact subjects showed no significant neural activation differences when imitating two dissimilar arm types. The equivalent left parietal and mesial frontal activity during planning in these subjects is characteristic of typical tool use pantomime activity (Goldenberg, 2003; Fridman et al., 2006; Goldenberg et al., 2007; Hermsdorfer et al., 2007; Tsuda et al., 2009). The activity over the primary motor and parietal cortex during execution is attributable to the demands of performing the motor task and accessing tool use related knowledge, respectively (Johnson-Frey, 2003; Glover, 2004; Wheaton et al., 2005a,c; Buxbaum et al., 2006; 


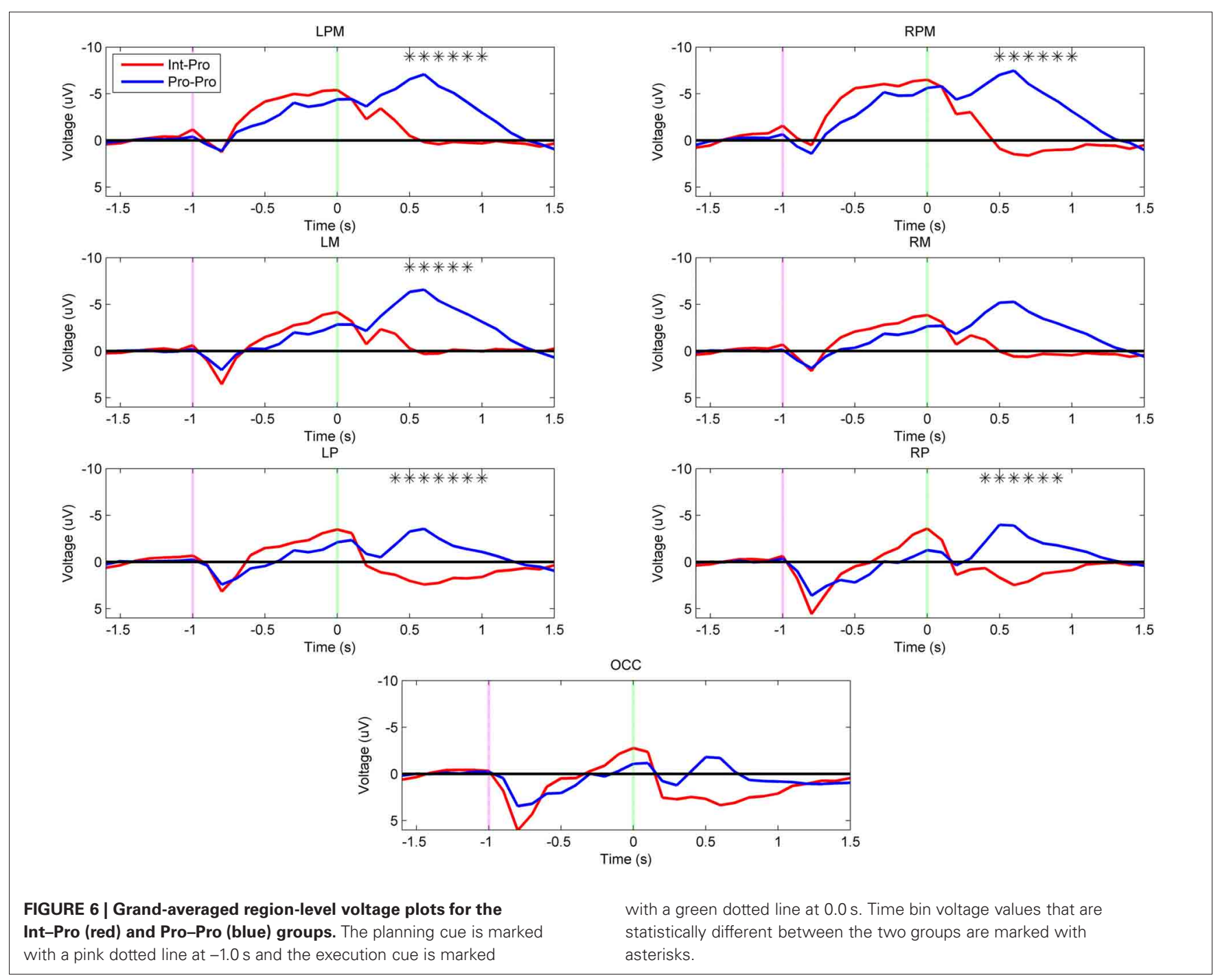

Creem-Regehr, 2009). These results suggest that intact subjects are not sensitive to neurobehavioral variations when imitating the highly dissimilar prosthetic limb. Movement planning and execution proceed with no statistical differences, regardless of the state of the arm viewed in the demonstration. We propose that for intact subjects, the task goal is the most salient aspect of the viewed action; observation of which will result in parietofrontal activation related to action encoding (Caggiano et al., 2011; Vingerhoets et al., 2011). Therefore, the normal left parietal, mesial frontal and motor cortex mechanisms are engaged to an equivalent extent for both conditions, despite the differences in conspecifics of the demonstration arm types. This result supports recent evidence describing the normal action encoding system as end-effector independent (Rochat et al., 2010; van Elk et al., 2011).

\section{ACTION PLANNING IN PROSTHESIS USERS}

\section{Prosthesis users imitating intact demonstrations}

The Pro-Int group showed increased right parietal and occipital positivity in addition to the typical parietofrontal negativity during movement planning compared to the Pro-Pro group. We argue that due to the mismatch of the subject's arm with that of the video demonstration, the planning phase can no longer proceed as normal. In this case, the movement observed in the demonstration of an intact limb may not solely engage the classical MNS (Rizzolatti and Craighero, 2004). It is proposed that this functional incongruity results in the increased visuospatial demand of the task and thus, requires additional occipitoparietal activity to facilitate task performance (Buccino et al., 2004).

There is precedence establishing that action encoding mechanisms may exist outside of the standard parietofrontal mirror neuron network. It has been proposed that the mentalizing system, which is important for understanding the intents of others, may be a candidate to serve this role when observing nonconspecific actions (Wheatley et al., 2007). Using this logic, the mentalizing system is engaged if no pre-existing motor template for the observed behavior exists (Van Overwalle and Baetens, 2009). In this case, enhanced visual comprehension is required to convert the non-conspecific action into one that can be completed successfully. Under the mentalizing model, amputees may 
be able to recognize the task goal, but are unable to quickly develop new motor patterns for controlling their prosthetic device and rely on additional visual mechanisms for planning the movement. Thus, when prosthesis users imitate an intact subject, the muscle onset order may be affected; potentially reflecting a diminished resonance for the action (Strafella and Paus, 2000; Funase et al., 2007; Alaerts et al., 2009). This mentalizing concept is corroborated by recent work demonstrating engagement of the mentalizing system in a congenital amputee when observing and imitating the actions of intact subjects (Aziz-Zadeh et al., 2012). Previous studies also suggest abnormal visuomotor processing demands for reaching tasks in amputees (Metzger et al., 2010). Presumably, this pattern of visuomotor conversion activity would continue until an appropriate motor template could be created (Mizelle et al., 2011). Investigation is ongoing to determine when and how the MNS may be engaged after such an update is completed.

\section{Prosthesis users imitating other prosthesis users}

Uniquely, when imitating prosthesis user demonstrations, there are no statistically significant differences between intact subjects and prosthesis users in right parietal or occipital areas during movement planning. This supports our proposal that the neural planning activations in a prosthesis user can resemble that of an intact subject, as long as there is an arm type match for the prosthesis user. According to the ideomotor principle, representations of actions are stored in the form of their effects. A bidirectional connection between an action and its effects is established through the association of one's own motor repertoires with the observed effects of the action (Hommel et al., 2001; Wohlschlager et al., 2003; Hommel, 2009). The motor repertoires of amputees may have been updated to include their prosthesis via the accumulation of experience observing the effects of actions performed with the device.

We propose that, due to the matching limb state, the prosthesis users' action representations are more strongly engaged by observing the actions of other prosthesis users. The unique cortical activity patterns reported in this study during imitation of a mismatched limb may be a result of the observer's motor repertoires converging towards the prosthetic limb, while diverging away from that of the amputated biological limb. This proposed divergence may be observed in the EMG onset results, which also suggest that the Pro-Pro (limb match) group converges to the intact limb pattern while the Pro-Int (limb mismatch) group exhibits a unique pattern. Recent work has demonstrated that the motor system is capable of incorporating non-biological components (tools) to complete a task while commonly activating the MNS structures responsible for use of the hand by itself (Umilta et al., 2008). As the prosthesis is integrated into the amputee motor repertoire, the matching prosthetic limb video demonstration may preferentially engage the parietofrontal activity typically seen in intact subjects with normal motor repertoires, thus decreasing the dependence on additional processing support from the parietooccipital regions.

The similarities between intact subjects and prosthesis users when imitating prosthesis users (Int-Pro vs. Pro-Pro) appear to be confined to the planning phase. Once in the movement execution phase, the prosthesis users generally show higher negativity than the intact subjects in bilateral premotor, left motor, and bilateral parietal areas. This effect is a focus of ongoing task performance studies using EMG and electrogoniometry.

\section{CLINICAL RELEVANCE}

These results have implications on the design and implementation of rehabilitation and occupational therapy protocols for amputees. Standard therapy involves training with an intact occupational therapist (Lake, 1997). Based on our findings, we propose that amputees may engage atypical planning mechanisms as they use their prosthetic device during any imitation-based therapy. This activity may be a reflection of the difficulty of the subject to easily translate the therapist's actions into their own actions.

It has been established that full functional adaptation of a prosthesis is not common; with $75 \%$ of amputees considering their devices to be primarily aesthetic while 33\% completely reject the device due to lack of perceived utility (Datta et al., 2004). Amputees cite lack of clear understanding of how to use their devices as well as dissatisfaction with the challenge of performing tasks of daily living (Kejlaa, 1993; Dudkiewicz et al., 2004). Amputees also encounter unique challenges in incorporating their prosthesis into tool use tasks due to altered sensory feedback (Ridding and Rothwell, 1995, 1997; Irlbacher et al., 2002; Reilly et al., 2008; Rosen et al., 2009; Gillespie et al., 2010; Stepp and Matsuoka, 2010; Rossini et al., 2011) and sense of agency (Ehrsson et al., 2008; Cipriani et al., 2009; Rosen et al., 2009). In addition to these difficulties, amputees report an uncomfortable foreignness when operating their prostheses (Smurr et al., 2008). It has been suggested that these challenges and the corresponding deviations in normal neural control strategies may influence the degree to which a patient successfully incorporates their device into activities of daily life (Cohen et al., 1991; Rossini et al., 2011). Further investigation is needed to determine if these occurrences are related to the atypical neural planning mechanisms presented in this work.

Our results suggest that while the neural activations of intact subjects seem to not differ when imitating movements performed by actors with different arm types, prosthesis users are susceptible to arm type. However, it is possible to elicit typical left parietofrontal cortical activation patterns in prosthesis users during imitation planning, provided they imitate other prosthesis users as opposed to intact actors. This effect may be accompanied by modification in the muscle activation patterns during execution of the movements. The current standard of rehabilitation for amputees may necessitate atypical planning mechanisms while learning to use their prosthetic device based on instruction by an intact therapist. A future direction of this work is to investigate whether or not the patterns of neural activation described here can inform the development of new methods for training prosthesis users, which may yield improved functional outcomes.

\section{ACKNOWLEDGMENTS}

We wish to thank J. C. Mizelle, Ph.D. for assistance in experimental design, data analysis, and results interpretation. This study was supported by the National Institutes of Health 2009 Pre-Doctoral Prosthetics and Orthotics Research Fellowship Program; National Institutes of Health National Center for Medical Rehabilitation Research T32 Award (\#5T32HD055180-03). 


\section{REFERENCES}

Alaerts, K., Swinnen, S. P., and Wenderoth, N. (2009). Is the human primary motor cortex activated by muscular or directiondependent features of observed movements? Cortex 45, 1148-1155.

Aziz-Zadeh, L., Sheng, T., Liew, S. L., and Damasio, H. (2012). Understanding otherness: the neural bases of action comprehension and pain empathy in a congenital amputee. Cereb. Cortex 22, 811-819.

Biddiss, E., and Chau, T. (2007a). Upper-limb prosthetics: critical factors in device abandonment. Am. J. Phys. Med. Rehabil. 86, 977-987.

Biddiss, E. A., and Chau, T. T. (2007b). Upper limb prosthesis use and abandonment: a survey of the last 25 years. Prosthet. Orthot. Int. 31, 236-257.

Bohlhalter, S., Hattori, N., Wheaton, L., Fridman, E., Shamim, E. A., Garraux, G., and Hallett, M. (2009). Gesture subtype-dependent left lateralization of praxis planning: an event-related fMRI study. Cereb. Cortex 19, 1256-1262.

Bouwsema, H., van Der Sluis, C. K., and Bongers, R. M. (2010). Movement characteristics of upper extremity prostheses during basic goal-directed tasks. Clin. Biomech. (Bristol, Avon) 25, 523-529.

Buccino, G., Binkofski, F., Fink, G. R., Fadiga, L., Fogassi, L., Gallese, V., Seitz, R. J., Zilles, K., Rizzolatti, G., and Freund, H. J. (2001). Action observation activates premotor and parietal areas in a somatotopic manner: an fMRI study. Eur. J. Neurosci. 13, 400-404.

Buccino, G., Lui, F., Canessa, N., Patteri, I., Lagravinese, G., Benuzzi, F., Porro, C. A., and Rizzolatti, G. (2004). Neural circuits involved in the recognition of actions performed by nonconspecifics: an FMRI study. J. Cogn. Neurosci. 16, 114-126.

Buxbaum, L. J., Kyle, K. M., Tang, K., and Detre, J. A. (2006). Neural substrates of knowledge of hand postures for object grasping and functional object use: evidence from fMRI. Brain Res. 1117, 175-185.

Caggiano, V., Fogassi, L., Rizzolatti, G., Pomper, J. K., Thier, P., Giese, M. A., and Casile, A. (2011). View-based encoding of actions in mirror neurons of area $\mathrm{f} 5$ in macaque premotor cortex. Curr. Biol. 21, 144-148.

Cattaneo, L., and Rizzolatti, G. (2009). The mirror neuron system. Arch. Neurol. 66, 557-560.

Cipriani, C., Antfolk, C., Balkenius, C. Rosen, B., Lundborg, G., Carrozza M. C., and Sebelius, F. (2009). A novel concept for a prosthetic hand with a bidirectional interface: a feasibility study. IEEE Trans. Biomed. Eng. 56, 2739-2743.

Cohen, L. G., Bandinelli, S., Findley, T. W., and Hallett, M. (1991). Motor reorganization after upper limb amputation in man. A study with focal magnetic stimulation. Brain 114(Pt 1B), 615-627.

Creem-Regehr, S. H. (2009). Sensorymotor and cognitive functions of the human posterior parietal cortex involved in manual actions. Neurobiol. Learn. Mem. 91, 166-171.

Datta, D., Selvarajah, K., and Davey, N. (2004). Functional outcome of patients with proximal upper limb deficiency-acquired and congenital. Clin. Rehabil. 18, 172-177.

Desmond, D. M., and Maclachlan, M. (2005). Factor structure of the Trinity Amputation and Prosthesis Experience Scales (TAPES) with individuals with acquired upper limb amputations. Am. J. Phys. Med. Rehabil. 84, 506-513.

Dudkiewicz, I., Gabrielov, R., SeivNer, I., Zelig, G., and Heim, M. (2004). Evaluation of prosthetic usage in upper limb amputees. Disabil. Rehabil. 26, 60-63.

Ehrsson, H. H., Rosen, B., Stockselius, A., Ragno, C., Kohler, P., and Lundborg, G. (2008). Upper limb amputees can be induced to experience a rubber hand as their own. Brain 131, 3443-3452.

Fridman, E. A., Immisch, I., Hanakawa, T., Bohlhalter, S., Waldvogel, D., Kansaku, K., Wheaton, L., Wu, T., and Hallett, M. (2006). The role of the dorsal stream for gesture production. Neuroimage 29, 417-428.

Funase, K., Tabira, T., Higashi, T. Liang, N., and Kasai, T. (2007). Increased corticospinal excitability during direct observation of selfmovement and indirect observation with a mirror box. Neurosci. Lett $419,108-112$.

Gillespie, R. B., Contreras-Vidal, J. L., Shewokis, P. A., O'malley, M. K., Brown, J. D., Agashe, H., Gentili, R., and Davis, A. (2010). Toward improved sensorimotor integration and learning using upper-limb prosthetic devices. Conf. Proc. IEEE Eng. Med. Biol. Soc. 2010, 5077-5080.

Glover, S. (2004). Separate visual representations in the planning and control of action. Behav. Brain Sci. 27, 3-24. discussion: 24-78.

Goldenberg, G. (2003). Pantomime of object use: a challenge to cerebral localization of cognitive function. Neuroimage 20 (Suppl. 1) S101-S106.

Goldenberg, G., Hermsdorfer, J., Glindemann, R., Rorden, C., and Karnath, H. O. (2007). Pantomime of tool use depends on integrity of left inferior frontal cortex. Cereb. Cortex 17, 2769-2776.

Hermsdorfer, J., Terlinden, G., Muhlau, M., Goldenberg, G., and Wohlschlager, A. M. (2007). Neura representations of pantomimed and actual tool use: evidence from an event-related fMRI study. Neuroimage 36 (Suppl. 2), T109-T118.

Hommel, B. (2009). Action control according to TEC (theory of event coding). Psychol. Res. 73, 512-526.

Hommel, B., Musseler, J., Aschersleben, G., and Prinz, W. (2001). The Theory of Event Coding (TEC): a framework for perception and action planning. Behav. Brain Sci. 24, 849-878. discussion: 878-937.

Irlbacher, K., Meyer, B. U., Voss, M., Brandt, S. A., and Roricht, S. (2002). Spatial reorganization of cortical motor output maps of stump muscles in human upperlimb amputees. Neurosci. Lett. 321 , 129-132.

Jackson, M. S. K. (2004). Pain after amputation. Contin. Educ. Anesth Crit. Care Pain 4, 20-23.

Jankelowitz, S. K., and Colebatch, J. G. (2002). Movement-related potentials associated with self-paced, cued and imagined arm movements. Exp. Brain Res. 147, 98-107.

Jeannerod, M., Arbib, M. A., Rizzolatti, G., and Sakata, H. (1995). Grasping objects - the cortical mechanisms of visuomotor transformation. Trends Neurosci. 18, 314-320.

Johnson-Frey, S. H. (2003). What's so special about human tool use? Neuron 39, 201-204.

Johnson-Frey, S. H. (2004). The neural bases of complex tool use in humans. Trends Cogn. Sci. 8, 71-78.

Karl, A., Diers, M., and Flor, H (2004a). P300-amplitudes in upper limb amputees with and without phantom limb pain in a visual oddball paradigm. Pain 110, 40-48.

Karl, A., Muhlnickel, W., Kurth, R., and Flor, H. (2004b). Neuroelectric source imaging of steady-state movement-related cortical potentials in human upper extremity amputees with and without phantom limb pain. Pain 110, 90-102.

Kejlaa, G. H. (1993). Consumer concerns and the functional value of prostheses to upper limb amputees. Prosthet. Orthot. Int. 17, 157-163.
Lake, C. (1997). Effects of prosthetic training on upper-extremity prosthesis use. J. Prosthet. Orthot. 9, $3-12$.

Losier, Y., Englehart, K., and Hudgins, B. (2011). Evaluation of shoulder complex motion-based input strategies for endpoint prosthetic-limb control using dual-task paradigm. J. Rehabil. Res. Dev. 48, 669-678.

Metzger, A. J., Dromerick, A. W. Schabowsky, C. N., Holley, R. J., Monroe, B., and Lum, P. S. (2010) Feedforward control strategies of subjects with transradial amputation in planar reaching. J. Rehabil. Res. Dev. 47, 201-211.

Mizelle, J. C., Tang, T., Pirouz, N. and Wheaton, L. A. (2011). Forming tool use representations: a neurophysiological investigation into tool exposure. J. Cogn. Neurosci. 23, 2920-2934.

Mizelle, J. C., and Wheaton, L. A. (2010). The neuroscience of storing and molding tool action concepts: how "plastic" is grounded cognition? Front. Cogn. 1:195. doi: 10.3389/fpsyg.2010.00195

Moll, J., De Oliveira-Souza, R., Passman, L. J., Cunha, F. C. Souza-Lima, F., and Andreiuolo, P. A. (2000). Functional MRI correlates of real and imagined tool-use pantomimes. Neurology 54 1331-1336.

Montoya, P., Ritter, K., Huse, E. Larbig, W., Braun, C., Topfner, S., Lutzenberger, W., Grodd, W. Flor, H., and Birbaumer, N. (1998). The cortical somatotopic map and phantom phenomena in subjects with congenital limb atrophy and traumatic amputees with phantom limb pain. Eur. J. Neurosci. 10, 1095-1102.

Oldfield, R. C. (1971). The assessment and analysis of handedness: the Edinburgh inventory. Neuropsychologia 9, 97-113.

Ortigue, S., Sinigaglia, C., Rizzolatti, G., and Grafton, S. T. (2010). Understanding actions of others: the electrodynamics of the left and right hemispheres. A high-density EEG neuroimaging study. PLOS ONE 5:e12160. doi: 10.1371/journal.pone.0012160

Quallo, M. M., Price, C. J., Ueno, K., Asamizuya, T., Cheng, K., Lemon, R. N., and Iriki, A. (2009). Gray and white matter changes associated with tool-use learning in macaque monkeys. Proc. Natl. Acad. Sci. U.S.A. 106, 18379-18384.

Ramachandran, V. S., and Hirstein, W. (1998). The perception of phantom limbs. The D. O. Hebb lecture. Brain 121(Pt 9), 1603-1630. 
Reilly, K. T., Schieber, M. H., and Mcnulty, P. A. (2008). Selectivity of voluntary finger flexion during ischemic nerve block of the hand. Exp. Brain Res. 188, 385-397.

Reinkensmeyer, D. J., Bonato, P., Boninger, M. L., Chan, L., Cowan, R. E., Fregly, B. J., and Rodgers, M. M. (2012). Major trends in mobility technology research and development: overview of the results of the NSF-WTEC European study. J. Neuroeng. Rehabil. 9, 22.

Ridding, M. C., and Rothwell, J. C. (1995). Reorganisation in human motor cortex. Can. J. Physiol. Pharmacol. 73, 218-222.

Ridding, M. C., and Rothwell, J. C. (1997). Stimulus/response curves as a method of measuring motor cortical excitability in man. Electroencephalogr. Clin. Neurophysiol. 105, 340-344.

Rizzolatti, G., and Craighero, L. (2004). The mirror-neuron system. Annu. Rev. Neurosci. 27, 169-192.

Rizzolatti, G., and Sinigaglia, C. (2010). The functional role of the parietofrontal mirror circuit: interpretations and misinterpretations. Nat. Rev. Neurosci. 11, 264-274.

Rochat, M. J., Caruana, F., Jezzini, A., Escola, L., Intskirveli, I., Grammont, F., Gallese, V., Rizzolatti, G., and Umilta, M. A. (2010). Responses of mirror neurons in area F5 to hand and tool grasping observation. Exp. Brain Res. 204, 605-616.

Rosen, B., Ehrsson, H. H., Antfolk, C., Cipriani, C., Sebelius, F., and Lundborg, G. (2009). Referral of sensation to an advanced humanoid robotic hand prosthesis. Scand. J. Plast. Reconstr. Surg. Hand Surg. 43, 260-266.

Rossini, P. M., Rigosa, J., Micera, S., Assenza, G., Rossini, L., and Ferreri, F. (2011). Stump nerve signals during transcranial magnetic motor cortex stimulation recorded in an amputee via longitudinal intrafascicular electrodes. Exp. Brain Res. 210, $1-11$.
Schabowsky, C. N., Dromerick, A. W., Holley, R. J., Monroe, B., and Lum, P. S. (2008). Trans-radial upper extremity amputees are capable of adapting to a novel dynamic environment. Exp. Brain Res. 188, 589-601.

Schaefer, M., Muhlnickel, W., Grusser, S. M., and Flor, H. (2002). Reliability and validity of neuroelectric source imaging in primary somatosensory cortex of human upper limb amputees. Brain Topogr. 15, 95-106.

Semlitsch, H. V., Anderer, P., Schuster, P., and Presslich, O. (1986). A solution for reliable and valid reduction of ocular artifacts, applied to the P300 ERP. Psychophysiology 23, 695-703.

Shimada, S. (2010). Deactivation in the sensorimotor area during observation of a human agent performing robotic actions. Brain $\operatorname{Cogn}$. 72, 394-399.

Smurr, L. M., Gulick, K., Yancosek, K., and Ganz, O. (2008). Managing the upper extremity amputee: a protocol for success. J. Hand Ther. 21, 160-175. quiz: 176.

Stepp, C. E., and Matsuoka, Y. (2010). Relative to direct haptic feedback, remote vibrotactile feedback improves but slows object manipulation. Conf. Proc. IEEE Eng. Med. Biol. Soc. 2010, 2089-2092.

Strafella, A. P., and Paus, T. (2000). Modulation of cortical excitability during action observation: a transcranial magnetic stimulation study. Neuroreport 11, 2289-2292.

Tai, Y. F., Scherfler, C., Brooks, D. J., Sawamoto, N., and Castiello, U. (2004). The human premotor cortex is "mirror" only for biological actions. Curr. Biol. 14, 117-120.

Tsuda, H., Aoki, T., Oku, N., Kimura, Y., Hatazawa, J., and Kinoshita, H. (2009). Functional brain areas associated with manipulation of a prehensile tool: a PET study. Hum. Brain Mapp. 30, 2879-2889.
Umilta, M. A., Escola, L., Intskirveli, I., Grammont, F., Rochat, M. Caruana, F., Jezzini, A., Gallese, V., and Rizzolatti, G. (2008). When pliers become fingers in the monkey motor system. Proc. Natl. Acad. Sci. U.S.A. 105, 2209-2213.

van Elk, M., van Schie, H. T., and Bekkering, H. (2011). Imitation of hand and tool actions is effectorindependent. Exp. Brain Res. 214 539-547.

Van Overwalle, F., and Baetens, K (2009). Understanding others' actions and goals by mirror and mentalizing systems: a meta-analysis. Neuroimage 48 , 564-584.

Velliste, M., Perel, S., Spalding, M. C., Whitford, A. S., and Schwartz, A. B. (2008). Cortical control of a prosthetic arm for self-feeding. Nature 453, 1098-1101.

Vingerhoets, G. (2008). Knowing about tools: neural correlates of tool familiarity and experience. Neuroimage 40, 1380-1391.

Vingerhoets, G., Vandekerckhove, E. Honore, P., Vandemaele, P., and Achten, E. (2011). Neural correlates of pantomiming familiar and unfamiliar tools: action semantics versus mechanical problem solving? Hum. Brain Mapp. 32, 905-918.

Wheatley, T., Milleville, S. C., and Martin, A. (2007). Understanding animate agents: distinct roles for the social network and mirror system. Psychol. Sci. 18, 469-474.

Wheaton, L. A., Bohlhalter, S., Nolte, G., Shibasaki, H., Hattori, N. Fridman, E., Vorbach, S., Grafman, J., and Hallett, M. (2008). Corticocortical networks in patients with ideomotor apraxia as revealed by EEG coherence analysis. Neurosci. Lett. 433, 87-92.

Wheaton, L., Fridman, E., Bohlhalter, S., Vorbach, S., and Hallett, M. (2009). Left parietal activation related to planning, executing and suppressing praxis hand movements. Clin. Neurophysiol. 120, 980-986.
Wheaton, L. A., Nolte, G., Bohlhalter, S., Fridman, E., and Hallett, $M$ (2005a). Synchronization of parietal and premotor areas during preparation and execution of praxis hand movements. Clin. Neurophysiol. 116, 1382-1390.

Wheaton, L. A., Shibasaki, H., and Hallett, M. (2005b). Temporal activation pattern of parietal and premotor areas related to praxis movements. Clin. Neurophysiol. 116, 1201-1212.

Wheaton, L. A., Yakota, S., and Hallett, M. (2005c). Posterior parietal negativity preceding self-paced praxis movements. Exp. Brain Res. 163, 535-539.

Wohlschlager, A., Gattis, M., and Bekkering, H. (2003). Action generation and action perception in imitation: an instance of the ideomotor principle. Philos. Trans. R. Soc. Lond. B Biol. Sci. 358 501-515.

Conflict of Interest Statement: The authors declare that the research was conducted in the absence of any commercial or financial relationships that could be construed as a potential conflict of interest.

Received: 13 March 2012; accepted: 01 June 2012; published online: 29 June 2012.

Citation: Cusack WF, Cope M, Nathanson S, Pirouz N, Kistenberg $R$ and Wheaton LA (2012) Neural activation differences in amputees during imitation of intact versus amputee movements. Front. Hum. Neurosci. 6:182. doi: 10.3389/fnhum.2012.00182 Copyright (c) 2012 Cusack, Cope Nathanson, Pirouz, Kistenberg and Wheaton. This is an open-access article distributed under the terms of the Creative Commons Attribution Non Commercial License, which permits non-commercial use, distribution, and reproduction in other forums, provided the original authors and source are credited. 


\section{APPENDIX}

Pantomime Recognition Scale

1. Movement is present, but difficult to decipher and prolonged with pauses.

2. Movement is recognizable, but with severe temporal and spatial errors.

3. Movement is fair, but with moderately prolonged movement sequences and temporal, spatial, and/or context errors.

4. Movement is error free. 